\title{
L'ÉPIGRAMME I, 109 DE MARTIAL : UN POÈME ENCOMIASTIQUE OU SATIRIQUE ?*
}

\begin{abstract}
RÉSUMÉ : L'épigramme I, 109 de Martial est-elle l'éloge convenu de l'animal favori d'un patron que l'on y a généralement lu ? Il nous semble, au contraire, que le génie satirique de Martial culmine dans cette pièce où l'auteur exploite en virtuose l'ambiguïté des formes non explicites de discours rapporté - mentions et discours au style direct libre - grâce auxquelles il se livre à un pastiche et à une mise à distance ironique des propos louangeurs du maître entiché de son animal de compagnie. Martial se rattache-t-il, en ce qui concerne ces animaux, à la tradition encomiastique ou à la tradition satirique ? Lui arrive-t-il de tourner en dérision des personnes réelles désignées par leur véritable nom ? Se permet-il, enfin, de porter sur ses protecteurs un regard critique ? Autant de questions de portée plus générale que met en jeu l'interprétation de cette épigramme.
\end{abstract}

SUMMARY : Is Martial's epigram 1.109 the encomium of a patron's pet dog, as it has been generally admitted ? It seems to us, on the contrary, that Martial's satirical genius reaches its highest point in this poem where the writer artistically plays with the ambiguous categories of reported discourse - free direct discourse and mentions - in order to write a pastiche of a master's praises for his pet which he presents with ironic distance. Are Martial's epigrams connected with the encomiastic or the satirical tradition concerning pets ? Does Martial sometimes mock real persons referred to by their own name ? And lastly, can he be critical of his patrons? Such are the issues of wider scope at stake in the interpretation of this epigram.

Issa est passere nequior Catulli. Issa est purior osculo columbae. Issa est blandior omnibus puellis. Issa est carior Indicis lapillis. Issa est deliciae catella Publi. Hanc tu, si queritur, loqui putabis ; sentit tristitiamque gaudiumque. Collo nixa cubat capitque somnos, ut suspiria nulla sentiantur ; et desiderio coacta uentris gutta pallia non fefellit ulla, sed blando pede suscitat toroque deponi monet et rogat leuari. Castae tantus inest pudor catellae, ignorat Venerem ; nec inuenimus dignum tam tenera uirum puella. Hanc ne lux rapiat suprema totam,
Issa est plus friponne que le moineau de Catulle. Issa est plus pure que le baiser de la colombe. Issa est plus caressante que toutes les jeunes femmes. Issa est plus précieuse que les joyaux de l'Inde.

5 Issa est la petite chienne qui fait les délices de Publius. Si elle gémit, tu croiras qu'elle parle ; elle ressent tristesse et joie.

Appuyée sur votre cou, elle repose et s'abandonne au sommeil sans faire entendre le moindre soupir.

10 Et, pressée par les besoins de son ventre, jamais, par aucune goutte, elle n'a trahi la couverture, mais, d'une patte caressante, elle vous éveille, demande à être descendue de la couche et à se soulager. Cette chaste petite chienne a tant de pudeur

15 qu'elle ignore Vénus; et nous ne trouvons pas de mari digne d'une si tendre jeune femme. Afin que son heure dernière ne l'emporte pas tout entière,

\footnotetext{
Nous aimerions exprimer toute notre gratitude aux membres du comité de la rédaction de la Revue de philologie, de littérature et d'histoire ancienne, et tout particulièrement à M. P. Flobert, pour les améliorations que leurs remarques nous ont permis d'apporter à notre texte, ainsi qu'à MM. É. Wolff, J. Trinquier et P.-V. Desarbres dont les suggestions et les encouragements nous ont été précieux.
} 
Frédérique Fleck, «L'épigramme I, 109 de Martial : un poème encomiastique ou satirique ? » Article paru dans la Revue de philologie 82/1, 2008 [2010], p. 49-75.

picta Publius exprimit tabella, in qua tam similem uidebis Issam, ut sit tam similis sibi nec ipsa. Issam denique pone cum tabella : aut utramque putabis esse ueram, aut utramque putabis esse pictam.
Publius en fait peindre un portrait, dans lequel tu verras une Issa si ressemblante

20 qu'elle-même ne se ressemble pas tant. Enfin, mets Issa à côté du portrait : ou tu croiras que tous deux sont vrais, ou tu croiras que tous deux sont peints. ${ }^{1}$

Le poème 109 du premier livre d'épigrammes de Martial, dont on vient de parcourir le texte, est le plus souvent lu comme un éloge de la chienne Issa ${ }^{2}$. On considère même qu'il pourrait s'agir d'une œuvre de commande : de même qu'il fait peindre son animal favori pour conserver son portrait après sa mort, Publius, le maître de la petite chienne, pourrait avoir demandé à Martial de composer pour lui une épigramme immortalisant Issa. Le poème s'inscrirait, toujours selon cette interprétation, dans la tradition de l'éloge d'un animal familier ${ }^{3}$.

Mario Citroni, dans son édition commentée du premier livre des Épigrammes, nuance toutefois ce point de vue : la disproportion entre le caractère dithyrambique de l'éloge et la modestie de son objet le conduit à percevoir dans le poème une certaine ironie, sans qu'il se résolve pourtant à considérer ce texte comme franchement satirique ${ }^{4}$. Si ce commentateur a bien senti que ce contraste induisait une distanciation et que certains passages étaient ambigus et pouvaient contenir des allusions érotiques, il n'a pas saisi, nous semble-t-il, le caractère essentiellement polyphonique de cette épigramme. Nous nous proposons de reprendre en détail l'étude de ce texte afin, d'abord, d'établir nettement le bien-fondé d'une lecture qui tienne compte de cette tonalité ironique dont plusieurs ouvrages, pourtant postérieurs à celui de M. Citroni, semblent ne faire aucun cas. Mais nous verrons aussi que l'hétérogénéité énonciative ne se manifeste pas seulement à travers les mentions ironiques: elle prend également la forme du pastiche. La reconnaissance de cette hétérogénéité amène à prendre

\footnotetext{
${ }^{1}$ Les traductions proposées sont personnelles, sauf indication contraire.

2 Ainsi, selon J. M. C. Toynbee, Animals in Roman Life and Art, Londres - Southampton, Thames and Hudson, 1973, p. 122, " the most delightful of all dog poems of the ancient Roman world is Martial's hendecasyllabic tribute to Issa ». P. Laurens, L'Abeille dans l'ambre. Célébration de l'épigramme, Paris, Les Belles Lettres, 1989, p. 309, parle de « l'évocation pittoresque de la petite chienne de Publius, (...) encadrée entre un prélude et un finale de caractère également laudatif: éloge d'Issa, éloge du portrait d'Issa exécuté par les soins de son maître.» J. P. Sullivan, Martial: the Unexpected Classic. A Literary and Historical Study, Cambridge, Cambridge University Press, 1991, p. 20, évoque l'épigramme en ces termes : «A poem (1.109) that could only have been written in pursuit of patronage follows almost immediately: it is an ingenious and humorous aretalogy on a pet lapdog Issa. »; la présence d'humour est ici relevée, mais l'épigramme est bien présentée comme appartenant au genre de l'éloge et visant à flatter Publius, interprétation qui s'accorde avec l'attitude bienveillante que l'on a coutume d'attribuer à l'humoriste. Plusieurs des imitations de cette épigramme citées par E.-T. Simon, Traduction en prose, et imitations en vers, nouvelles et complètes, avec texte latin en regard, des Épigrammes de M. Val. Martial ; enrichie de notes, et des meilleures imitations en vers français, depuis Clément Marot jusqu'à nos jours, Paris, F. Guitel, 1819, vol. 3, p. 384-395, témoignent d'une interprétation semblable ; nous aurons l'occasion d'y revenir.

${ }^{3}$ P. Howell, A Commentary on Book One of the Epigrams of Martial, Londres, The Athlone Press, 1980, p. 334 : «Naturally pets often formed subjects for poets, either to be praised in life (as here), or - much more commonly - to be lamented after death.» P. Richard, Les Épigrammes de Martial, Paris, Garnier, 1931 : « Martial célèbre la petite chienne de son ami Publius, comme Catulle avait fait l'éloge du moineau de Lesbie (Élégies 2), Ovide celui du perroquet de Corinne (Amours II, 6), Stace celui du perroquet de Melior (Silves II, 4) et Stella de la colombe d'Astérie [sic]. Il semble lui-même insinuer (v. 1 et 2) qu'il veut lutter contre le premier et le dernier de ces poèmes. »

${ }^{4}$ M. Citroni, M. Valerii Martialis epigrammaton liber primus, Florence, La nuova Italia, 1975, p. 333 : «la stessa elegante stilizzazione impedisce che l'ironia, da gioco festoso, diventi satira, e salva quindi, pur sotto un velo ironico, la funzione cortese dell'elogio ».
} 
Frédérique Fleck, «L'épigramme I, 109 de Martial : un poème encomiastique ou satirique ? »

Article paru dans la Revue de philologie 82/1, 2008 [2010], p. 49-75.

acte de la distanciation critique de l'épigrammatiste par rapport aux propos mentionnés qui en est constitutive, ainsi que du caractère d'attaque ad hominem conféré à l'ironie et au pastiche par le fait que ces propos peuvent être rapportés à une source bien identifiable.

Nous montrerons, en premier lieu, que ce poème s'inscrit mal dans le paradigme de l'éloge d'un animal familier dans lequel on veut le faire entrer, et que l'on trouve dans l'œuvre de Martial de nets témoignages de son attitude critique envers les maîtres trop entichés de leurs animaux de compagnie. Nous relèverons ensuite les différents types de dissonances qui fondent une lecture distanciée et nous les interpréterons en les replaçant dans le cadre d'un discours polyphonique qui invite à considérer ce poème comme une véritable satire $^{5}$. Cette étude de l'épigramme I, 109 nous amènera à prendre position dans le débat qu'a suscité l'œuvre panégyrique de Martial : le poète s'adonne-t-il à de plates flatteries envers l'empereur Domitien et un certain nombre de patrons dont il espère recevoir des subsides, ou bien exprime-t-il, de manière voilée, des critiques à l'encontre de ces riches protecteurs ? ${ }^{6}$

\section{Un poème encomiastique?}

L'inscription du poème I, 109 de Martial dans le paradigme de l'éloge d'un animal familier est délicate. Ces éloges se rencontrent le plus souvent sous la forme d'épicèdes tels que le poème 3 de Catulle sur la mort du moineau de Lesbie et les pièces qu'il a inspirées ${ }^{7}$ : celle d'Ovide sur le perroquet de Corinne (Amours II, 6) et celle de Stace sur le perroquet d'Atedius Melior (Silves II, 4) - lequel s'inspire d'ailleurs plus du poème d'Ovide que de celui de Catulle -, l'éloge épigraphique de la chienne Myia ( $C E$ 1512), ou encore le poème de Stella, ami de Martial, sur la colombe de son amie Ianthis ${ }^{8}$. Tel n'est pas le cas, en revanche, du poème de Martial puisqu'Issa, la chienne dont il est question, est vivante. Sa mort est bien évoquée au v. 17, mais Issa n'est ni décrite comme morte, ni imaginée comme

\footnotetext{
5 L'acception que nous retenons ici pour la notion de satire est celle, communément admise, de critique moqueuse destinée à corriger les mœurs par le rire.

${ }^{6}$ La première thèse constitue le point de vue traditionnel et a été reprise récemment, avec de nouveaux arguments, par Fr. Römer, « Mode und Methode in der Deutung panegyrischer Dichtung der nachaugusteischen Zeit », Hermes, 22, 1994, p. 95-113. La seconde a été défendue notamment par J. Garthwaite, « Martial, Book 6, on Domitian's Moral Censorship », Prudentia, 22, 1990, p. 13-22 et par N. Holzberg, Martial und das antike Epigramm, Darmstadt, Wissenschaftliche Buchgesellschaft, 2002 (voir p. 63-85). Pour un résumé du débat, voir S. Lorenz, Erotik und Panegyrik. Martials epigrammatische Kaiser, Tübingen, Narr, 2002, p. 45-48. É. Wolff, « Ambiguïtés de Martial», dans Actes du colloque "Le poète irrévérencieux », Lyon, ENS-LSH, 19-20 octobre 2007, à paraître, considère pour sa part que ces textes permettent une double lecture.

${ }^{7}$ Certains passages de ces poèmes, emblématiques de la tradition de l'épigramme encomiastique à Rome, ont pu être considérés comme parodiques, parfois même comme humoristiques ou ironiques. Voir, entre autres, G. Herrlinger, Totenklage um Tiere in der antiken Dichtung, Stuttgart, W. Kohlhammer, 1930, p. 75-90 ; A. Ronconi, Studi catulliani, Brescia, Paideia, 1971, p. 180 (première édition : 1953) ; M. Citroni, Poesia e lettori in Roma antica. Forme della comunicazione letteraria, Rome, Laterza, 1995, p. 120-127 ; D. F. S. Thomson, Catullus, Toronto - Buffalo - Londres, University of Toronto Press, 1997, p. 207-212 ; B. Weiden Boyd, « The Death of Corinna's Parrot Reconsidered : Poetry and Ovid's Amores », CJ, 82/3, 1987, p. 199-207 ; L. Cahoon, «In Response to Barbara Weiden Boyd, 'The Death of Corinna's Parrot Reconsidered : Poetry and Ovid's Amores' CJ, 82 (1987) 199-207. Psittacus Redux : Boyd's Bird and Mine (Or, Some Thoughts on Aims and Methods in Literary Studies)», CJ, 86/4, 1991, p. 368-376; K. R. Walters, "Catullan Echoes in the Second Century AD, $C E L 1512 », C W, 69,1976$, p. 353-359. Nous ne pouvons nous arrêter ici sur la question de leur interprétation; nous reviendrons ailleurs sur celle du carmen 3 de Catulle et sur les rapports qu'entretient avec ce texte l'épigramme I, 109 de Martial.

${ }^{8}$ Voir Martial I, 7 et VII, 14 où il est question de ce poème de Stella, qui ne nous a pas été conservé.
} 
Frédérique Fleck, «L'épigramme I, 109 de Martial : un poème encomiastique ou satirique ? »

Article paru dans la Revue de philologie 82/1, 2008 [2010], p. 49-75.

telle. Le poème de Martial se démarque donc déjà thématiquement de ces textes par le fait que l'animal familier n'est pas mort ${ }^{9}$.

Il s'en sépare encore plus nettement sur le plan de l'énonciation. Les épitaphes présentent tantôt des énoncés à la première personne du singulier dont l'énonciateur est l'animal défunt (ainsi en $C E 1175$ ou dans l'épigramme XI, 69 de Martial, dans lesquels ce sont respectivement les chiennes Margarita et Lydia qui s'expriment pour raconter leur existence et les circonstances de leur mort), tantôt des passages à la deuxième personne, souvent accompagnée de vocatifs, qui s'adressent à l'animal (ainsi en $C E$ 1176, où le maître de Patrice s'adresse à sa défunte chienne, et en $C E 1512$ où Myia est regrettée par l'amant de sa maîtresse). Les poèmes de Catulle et de ses imitateurs comportent de nombreuses apostrophes adressées à l'animal lui-même, mais aussi à d'autres êtres qui sont pris à témoins et invités à prendre le deuil, ou parfois encore au maître de l'animal. Rien de tel chez Martial : Issa et Publius sont toujours évoqués à la troisième personne ${ }^{10}$. Cette distance est aussi sensible dans le fait que l'animal n'appartient pas à la maîtresse du poète (comme chez Catulle, chez Ovide, chez Stella, ou dans l'épigramme anonyme sur la chienne Myia) ou au poète lui-même (comme dans l'épitaphe de la chienne Patrice, $C E$ 1176), mais à l'un de ses amis $^{11}$.

S'il est utile et pertinent de rapprocher le poème de Martial de son modèle catullien et, plus généralement, des textes appartenant à la tradition des poèmes funéraires littéraires et épigraphiques, il ne faut donc pas perdre de vue qu'il s'en distingue à la fois par son développement thématique et par ses particularités énonciatives ; 1'y assimiler purement et simplement risque d'en dénaturer le sens et la portée.

Par ailleurs, au vu de ce que l'on trouve dans le reste de l'œuvre de Martial, il parait peu probable que cet auteur ait pu se livrer sincèrement à l'éloge d'un animal de compagnie. M. Citroni ${ }^{12}$ a bien rappelé l'existence de deux traditions concernant les animaux familiers : la première, qui est d'abord représentée dans les épigrammes hellénistique, est celle de la célébration, tandis que la seconde, elle, condamne l'attachement excessif des maîtres à leurs animaux. D'après M. Citroni, les deux traditions coexistent dans l'œuvre de Martial. La tradition satirique y est représentée par le poème VII, 87, dans lequel Martial se moque ouvertement de l'affection que plusieurs de ses amis (parmi lesquels Publius apparaît justement) portent à leur animal favori et qui se termine par ce vers : qui uidet haec dominis monstra placere suis ( " celui qui voit ces monstres faire les délices de leurs maîtres »). Elle l'est aussi par le poème I, 83, dans lequel Martial tire une conclusion peu flatteuse des marques d'affection qu'un chien prodigue à sa maîtresse ${ }^{13}$. La tradition encomiastique serait illustrée par l'épigramme 69 du livre XI, épitaphe d'un chien de chasse. La défunte chienne Lydia y fait son propre éloge, en empruntant au style épique plusieurs comparaisons. Mais il faut noter que, si le chien de chasse est un animal domestique, il n'est pas un animal de compagnie ; les services qu'il rend légitiment l'attachement que son maître peut éprouver pour lui : cet attachement ne peut, dès lors, être considéré comme une marque de mollesse et

\footnotetext{
${ }^{9}$ Cette situation pourrait renvoyer, en revanche, au poème 2 de Catulle qui évoque les jeux du moineau avec sa maîtresse.

${ }^{10}$ Ces écarts de l'épigramme de Martial par rapport au modèle catullien font l'objet de corrections révélatrices dans certaines adaptations. Ainsi, dans le poème de T. S. Des Accords, cité par E.-T. Simon, 1819, vol. 3, p. 393394, la chienne appartient à la maîtresse du poète, et la pièce est entièrement adressée à l'animal.

${ }^{11}$ Tel est toutefois le cas chez Stace également.

12 1975, p. 332-333. On se reportera à cet ouvrage pour trouver des renvois précis aux textes représentant les deux traditions.

${ }^{13}$ Os et labra tibi lingit, Manneia, catellus : / non miror, merdas si libet esse cani. «Ton petit chien te lèche le visage et les lèvres, Manneia : je ne m’étonne pas que ce chien aime manger de la merde ! »
} 
de décadence, ni faire l'objet de critiques et de satires ${ }^{14}$. Ce poème ne constitue donc pas, à notre avis, la preuve de l'inscription, même occasionnelle, de Martial dans la tradition hellénistique des épigrammes sur les animaux. M. Citroni mentionne encore le poème IV, 32, mais il s'agit de la description d'une abeille prise dans une goutte d'ambre, ce qui nous éloigne tout à fait de la question des animaux de compagnie et ne saurait être allégué ici.

La tradition encomiastique concernant les animaux de compagnie n'est donc, à ce qu'il nous semble, pas représentée chez Martial, en dehors du cas, que nous discutons, de l'épigramme I, 109 sur la chienne Issa. Bien qu'il en souligne l'ironie, M. Citroni place ce poème dans la catégorie des éloges. Mais, pour notre part, nous considérons que, s'il se réfère bien à cette tradition encomiastique (nous verrons plus en détail de quelle manière dans la suite de notre étude), c'est à la tradition satirique que ce poème de Martial appartient en réalité, et c'est ce que nous nous proposons de montrer ${ }^{15}$. Contentons-nous de remarquer pour l'instant que cette lecture est a priori la plus indiquée, puisque seule la tradition satirique se trouve clairement attestée dans l'œuvre de Martial, par le poème VII, 87 notamment, épigramme satirique qui de surcroît mentionne précisément Publius et sa chienne. L'interprétation du poème comme une critique moqueuse est aussi plus en conformité avec le ton badin des pièces en hendécasyllabes phaléciens. Notons encore au passage que ce poème VII, 87 montre également qu'il arrive à Martial de faire la satire de ses amis ${ }^{16}$ - une satire certes mesurée en regard de la violence avec laquelle elle s'exprime dans d'autres épigrammes -, ce qui dément, ou du moins amène à nuancer, la thèse selon laquelle les noms des personnages fustigés dans les épigrammes seraient toujours fictifs ${ }^{17}$.

\section{Peinture d'Issa}

\subsection{Portrait d'Issa en fille de Publius}

La manière dont sont présentés Issa et ses rapports avec Publius est, à bien des égards, ambiguë. On apprend bien, au vers 5, qu'Issa est une petite chienne, catella, mais sans cette indication, reprise au vers 14 , et la comparaison du vers 1 avec le moineau de Catulle, nous

\footnotetext{
${ }^{14}$ Notons que dans cette épigramme XI, 69 rien n'évoque une familiarité excessive entre l'animal et son propriétaire : pas de jeux en commun, pas de scène de repos dans les bras ou sur le lit du maître.

${ }^{15}$ Notons que Pommereul, cité par E.-T. Simon, 1819, vol. 3, p. 384, a senti la difficulté que pouvait soulever cette louange d'un animal de compagnie et a entrepris de la lever en insérant dans son adaptation de l'épigramme, entre ce qui correspond au vers 5 et au vers 6 du texte latin, la justification suivante : "On peut, sans en rougir, l'aimer à la folie. / Aussi de Publius ne suis-je point surpris / que Fidèle ait captivé la tendresse. / N'appelons pas ce sentiment faiblesse, / l'attachement d'une chienne a son prix. / Est-il toujours plus sûr celui d'une maîtresse ? / J'en doute un peu : celui de Fidèle est certain. » (v. 7-13).

${ }^{16}$ On n'a que peu de renseignements sur le personnage de Publius, qui apparaît également dans les épigrammes II, 57, VII, 72 et VII, 87 ; il s'agit vraisemblablement d'un patron, mais avec lequel Martial pourrait avoir entretenu, comme avec Arruntius Stella - le patronus amicus par excellence -, des relations d'amitié (son nom est accompagné, en II, 57, du possessif meus qui marque un lien personnel). Voir D. Vallat, Onomastique, culture et société dans les Épigrammes de Martial, Bruxelles, Latomus, 2008 (p. 69 à propos de Publius et p. 6667 à propos de Stella).

${ }^{17}$ C'est la thèse exposée par A. Cartault, «Sur un emploi particulier des noms propres dans les épigrammes de Martial », Mélanges Boissier, Paris, A. Fontemoing, 1903, p. 103-113, qui distingue trois types d'épigrammes, les «pièces satiriques », les «pièces de confidences personnelles» et les «pièces honorifiques ». Selon cet auteur, concernant la première catégorie, Martial «a déclaré à diverses reprises qu'il n'entendait désigner personne en particulier et nous devons le croire jusqu'à plus ample informé » (p. 103).
} 
nous imaginerions sans doute bien autre chose ${ }^{18}$. Le moineau de Lesbie donne des morsures $(2,4)$, sautille et pépie (circumsiliens, 3, 9, pipiabat, 3, 10), la chienne Lydia est une chasseresse qui parcourt les forêts et combat les sangliers (Martial XI, 69), Margarita et Myia (CE 1175 et 1512) aboient, et la seconde accueille l'ami de sa maitresse en sautant autour de lui et en le mordillant; même dans le poème épigraphique funéraire pour la chienne Patrice, dans lequel l'humanisation de l'animal est la plus poussée ${ }^{19}$, l'attitude de Patrice à table est bien celle d'un chien, et elle donne des coups de langue et accueille son maître en remuant la queue. Rien de tel pour Issa - dont le nom est d'ailleurs un anthroponyme ${ }^{20}-$, en dehors du passage des vers 10 à 13 qui évoque en termes contournés sa propreté et contient la mention blando pede suscitat, " elle vous éveille d'une patte caressante » (le terme latin pes ne réfère toutefois pas spécifiquement aux animaux). Ses actions sont rapportées, dans le reste du texte et dans ce passage même, comme celles d'un être humain : queritur (6), sentit (7), monet et rogat (13), et plusieurs des termes qui la qualifient s'appliqueraient mieux à une jeune fille : purior (2), castae et pudor (14). Elle est d'ailleurs comparée à des jeunes femmes au vers 3 et finalement assimilée à une puella (16), à laquelle on cherche un mari (uirum). Ainsi, c'est comme la fille même de Publius qu'elle est présentée dans certains passages, notamment dans les vers 14 à 16 - ce qui n'est pas sans rappeler les appellatifs dont les maîtres affublent parfois leurs animaux domestiques («mon bébé », « ma fille ») ${ }^{21}$. Nous rencontrons là une première dissonance : est-il raisonnable de mettre au compte de Martial une humanisation si poussée de l'animal?

\subsection{Issa, huitième merveille du monde}

Il faut signaler encore une autre dissonance par rapport à la tonalité habituelle des écrits de Martial ; il s'agit de l'admiration naïvement enthousiaste pour Issa. Cette admiration béate s'exprime dès les quatre premiers vers qui forment une sorte d'arétalogie et dans lesquels le choix du comparant, qui représente à chaque fois le parangon de la qualité évoquée, donne au comparatif de supériorité la valeur d'un superlatif absolu (c'est le type melle dulcior). L'idée qu'Issa est quasiment capable de parler et qu'en tout cas elle ressent tout à la manière d'un être humain (6-7) témoigne de la même attitude. Celle-ci est encore particulièrement manifeste dans les derniers vers (19-23), à propos cette fois non plus d'Issa elle-même, mais du tableau qui en est fait, comme si tout ce qui touchait de près ou de loin à la chienne se trouvait, comme elle, magnifié : la peinture est, nous dit-on, si réussie qu'on ne peut discerner aucune différence entre la chienne et sa représentation, qui paraît aussi vivante

\footnotetext{
${ }^{18}$ M. Citroni, 1975, p. 334, note à propos du début du poème: «Si noterà in questi primi versi la sottile ambiguità erotica : anzi con procedimento che ricorda l'aprosdokèton epigrammatico, solo nel $v$. 5 M. rivela che non sta celebrando il delicato erotismo di una fanciulla, ma l'amabilità di una catella. »

${ }^{19}$ L'usage de la comparaison (v. $7: \operatorname{morib}(u s)$ argutis hominem similare paratam, « toi dont l'intelligence pouvait te faire l'égale d'un être humain ») maintient cependant une distance qu'on ne retrouve pas dans le poème de Martial où l'assimilation est directe.

${ }^{20}$ Ipsa, prononcé Issa, est employé couramment pour désigner la maîtresse. Voir CIL VIII, 8016.

${ }^{21}$ Pour une dénonciation de cette tendance à assimiler les animaux à des enfants, voir l'anecdote rapportée par

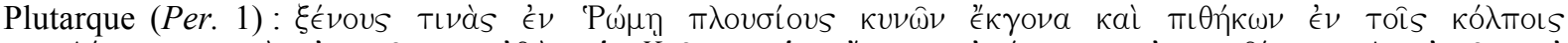

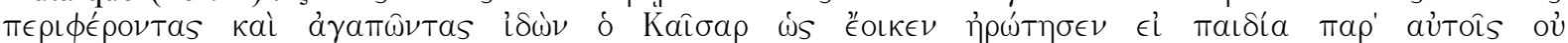

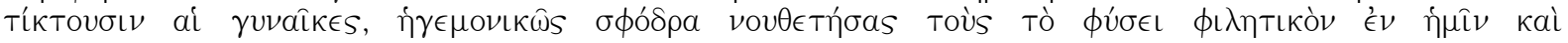

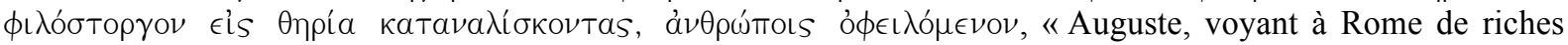
étrangers qui, en se promenant, portaient dans leurs bras et caressaient des jeunes chiens ou des petits singes, leur demanda, dit-on, si dans leur pays les femmes ne leur donnaient pas d'enfants. C'était là une manière tout impériale de blâmer ceux qui dépensent sur des bêtes notre sentiment naturel d'amour et d'affection qui est dû aux hommes » (trad. R. Flacelière et E. Chambry, C.U.F.).
} 
Frédérique Fleck, «L'épigramme I, 109 de Martial : un poème encomiastique ou satirique ? » Article paru dans la Revue de philologie 82/1, 2008 [2010], p. 49-75.

qu'elle. Dans le Satyricon (29, 1-2), ce n'est que parce qu'il est distrait par le spectacle qu'offre la demeure de Trimalchion qu'Encolpe se laisse terroriser par un molosse peint, et il est alors la risée de ses compagnons. Le motif de la peinture qui paraît plus vraie que nature se trouve chez Pline l'Ancien, mais en deux endroits seulement, et pour caractériser le travail de deux artistes qui se distinguent par la qualité tout à fait exceptionnelle des représentations qu'ils produisent ${ }^{22}$. L'épisode le plus développé concerne le peintre Parrhasios, qui défie son contemporain et émule Zeuxis ; comme on sait, Zeuxis réussit à tromper les oiseaux avec la représentation d'une grappe de raisins, ce qui est considéré comme une véritable prouesse, si bien qu'il se sent sûr d'avoir gagné le concours ; mais il est surpassé par Parrhasios, qui lui fait prendre pour réel un voile qu'il a représenté drapant son propre tableau et parvient donc, comble de l'art, à tromper non des animaux, mais un être humain et, qui plus est, un artiste même. Chez Martial, le motif est repris à propos de sculptures ou de motifs ciselés sur une coupe, œuvres de Phidias (III, 35) ou de Mentor (III, 40), ou que l'on pourrait égaler à celles de Phidias et même d'Athéna (VI, 13, pièce destinée à flatter Domitien) ${ }^{23}$. L'attribution d'une réussite semblable à un artiste obscur ${ }^{24}$, dont le nom n'est en tout cas pas assez célèbre pour mériter d'être mentionné, paraît donc tout à fait disproportionnée ${ }^{25}$.

L'admiration s'étend également aux moindres actions de la petite chienne, y compris les moins nobles : le fait qu'Issa ne fait pas ses besoins n'importe où se trouve ainsi évoqué. On peut déjà s'étonner que ces réalités triviales trouvent place dans le poème, mais on ne peut surtout manquer d'être frappé par la place qu'elles y occupent (pas moins de quatre vers sur un total de vingt-trois). Le décalage entre le style choisi et contourné qui est employé dans ces vers 10 à 13 et la réalité qui est décrite est ici proprement comique : l'urine n'est évoquée qu'à travers le mot gutta, sans autre précision, l'idée de vessie est difficilement perceptible dans la désignation métonymique qui en est faite (desiderio coacta uentris, 10), l'emploi du verbe fefellit (11) pour décrire l'action de souiller avec du liquide est pour le moins atypique et celui de leuari (13) est suffisamment obscur pour qu'il y ait lieu de se demander s'il signifie «se soulager» (passif à sens réfléchi) ${ }^{26}$ ou « être soulevé » pour revenir sur le lit (avec alors ellipse de l'action même de faire ses besoins) ${ }^{27}$. Dans ce passage, le style hérö̈-

\footnotetext{
${ }^{22}$ Pline l'Ancien, Histoire naturelle XXXV, 65 (à propos de l'un des tableaux de Parrhasius) et 155 (à propos de modelages de fruits et de poissons exécutés par Chalcosthénès).

${ }^{23}$ Artis Phidiacae toreuma clarum / pisces aspicis : adde aquam, natabunt. (III, 35), « Tu vois ces poissons, ce célèbre relief ciselé, œuvre de Phidias : mest-y de l'eau et ils nageront. »; Inserta phialae Mentoris manu ducta / lacerta uiuit et timetur argentum. (III, 40), «Ciselé sur la coupe où l'a représenté la main de Mentor, ce lézard est vivant et la crainte nous saisit devant cette pièce d'argenterie. »; Quis te Phidiaco formatam, Iulia, caelo, / uel quis Palladiae non putet artis opus ? / Candida non tacita respondet imagine lygdos / et placido fulget uiuus in ore decor. (VI, 13, 1-4), «Qui ne croirait pas, Julia, que c'est le ciseau de Phidias qui t'a sculptée ou que tu es l'œuvre de Pallas? Le marbre blanc nous répond par ce portrait parlant et une beauté vivante illumine ton doux visage. $\gg$

${ }^{24} \mathrm{Ou}$ à Publius lui-même, si l'on ne donne pas de valeur factitive au verbe exprimit (v. 18); c'est l'interprétation retenue par P. Laurens, 1989, p. 309.

${ }^{25}$ M. Citroni, 1975 , p. 340, qui ne note pas ce caractère outré, pense que le motif du portrait qui ne peut être distingué de son objet "verosimilmente era già in epigr. greci sui ritratti», mais est cependant amené à reconnaître : «non mi pare però che ci siano conservati esempi se non in un epigrammista molto tardo ».

${ }^{26}$ Le terme est ainsi traduit par H.-J. Izaac, Martial, Épigrammes, Paris, Les Belles Lettres, 1930-1933 : « et demande à se soulager ». Cet emploi du verbe serait à rapprocher, comme l'indique M. Citroni, 1975, p. 339, du passage de l'Histoire Auguste, Carac. 7 : leuandae uesicae gratia, " pour soulager sa vessie ».

${ }^{27}$ C'est le sens retenu par D. R. Schackleton Bailey, Martial, Epigrams, Cambridge - Londres, Loeb Classical Library, 1993 (« and then asks to be taken up »), P. Barié et W. Schindler, M. Valerius Martialis. Epigramme, Düsseldorf - Zürich, Artemis \& Winkler, 1999 ("und bittet, daß man sie dann wieder hochnehme ») entre autres.
} 
Frédérique Fleck, «L'épigramme I, 109 de Martial : un poème encomiastique ou satirique ? »

Article paru dans la Revue de philologie 82/1, 2008 [2010], p. 49-75.

comique trouve son expression la plus frappante ${ }^{28}$, mais ce style est mis en œuvre, de manière plus discrète, dans l'ensemble du poème ${ }^{29}$. Il l'est avec les différents termes que nous avons déjà relevés et qui assimilent Issa à une jeune femme, mais aussi avec une expression choisie comme capit somnos (8) au lieu du simple dormit, avec l'emploi métonymique de Venerem (15) pour désigner l'activité sexuelle, ou encore avec la périphrase lux suprema (17) que l'on trouve pour désigner la mort dans les poèmes épiques et tragiques ${ }^{30}$.

L'admiration enthousiaste manifestée pour Issa et pour tout ce qui touche à elle est si outrée, le vocabulaire et les tours employés pour la décrire relèvent d'un style si peu adapté à la trivialité du sujet qu'il paraît difficile de s'en tenir à une interprétation simple et naïve.

\subsection{Portrait d'Issa en maîtresse de Publius}

Cette impression est encore renforcée par ce que le texte suggère de la relation entre Publius et Issa et qui est, cette fois, tout a fait dans la veine de Martial. L'adjectif nequior, employé au vers 1, tout comme le substantif nequitia qui en est dérivé, prend souvent chez cet auteur des connotations sexuelles: dans l'épigramme III, 69, à côté des jeunes filles complaisantes et du vieillard que taquine encore une maîtresse, sont ainsi évoqués comme composant le lectorat des œuvres de Martial les nequam iuuenes (5); on pourra citer aussi l'épigramme IX, 67, qui commence par ces vers :

\section{Lasciuam tota possedi nocte puellam,}

cuius nequitias uincere nemo potest. ${ }^{31}$

Le terme est en outre employé à propos du moineau de Lesbie en VII, 14, 4 (Lesbia, nequitiis passeris orba $s u i^{32}$ ); or la fin de l'épigramme XI, 6 suggère que Martial faisait du poème de Catulle une lecture érotique, donnant au mot passer un double sens obscène :

Da nunc basia, sed Catulliana :

quae si tot fuerint quot ille dixit,

donabo tibi passerem Catulli. ${ }^{33}$

Cette première caractérisation, au seuil du poème, Issa est passere nequior Catulli, introduit donc en contrebande l'idée d'une relation entre Issa et Publius qui serait de nature érotique.

\footnotetext{
${ }^{28}$ Ce style héroï-comique ne se retrouve pas, en revanche, chez Du Bellay, qui intègre, en le transposant, dans son poème sur le petit chien Peloton, Divers Jeux rustiques, poème 27, «Épitaphe d'un petit chien », 53-58, ce passage de l'épigramme de Martial : «Et ne souilloit point sa couche / du ventre ny de la bouche, / car sans cesse il gratignoit / quand ce désir le poingnoit : / tant fut la petite beste / en toutes choses honneste. »

${ }^{29}$ Une semblable disproportion peut être signalée entre la taille de l'animal et la longueur du poème qui est consacré à l'évocation de ses vertus, comme l'indique M. Citroni, 1975, p. 331-332; c'est d'ailleurs ce que semble souligner Martial lui-même dans l'un des textes des Apophoreta (XIV, 198) : Delicias paruae si uis audire catellae, / narranti breuis est pagina tota mihi, « Si tu veux connaître les délices de cette toute petite chienne, c'est trop peu pour moi d'une page entière pour te les raconter ».

${ }^{30}$ Cf. Accius, $T r .37$ (= 296 D.) ; Homerus Latinus 946 ; Silius Italicus, Punica XV, 66.

31 «J'ai possédé toute la nuit une fille lascive, avec laquelle personne ne peut rivaliser en matière de dissipation. »

32 «Lesbie, privée des friponneries de son moineau ».

33 «Et maintenant, donne-moi des baisers, des baisers catulliens ! S'ils sont aussi nombreux qu'il l'a dit, je te donnerai le moineau de Catulle. » L'interprétation de ce poème et d'autres où il est question du passer Catulli est toutefois discutée. En faveur d'un sens obscène du mot passer, voir notamment Y. Nadeau, « Catullus' Sparrow, Martial, Juvenal and Ovid», Latomus, 43, 1984, p. 861-868, voir p. 863, et R. W. Hooper, «In Defence of Catullus' Dirty Sparrow », G\&R, 32, 1985, p. 162-178, voir plus précisément p. 169-170; ces vues sont contestées entre autres par J. N. Adams, The Latin Sexual Vocabulary, Londres, Duckworth, 1982, p. 32-33. Pour une interprétation différente, voir par exemple J. Ward Jones Jr, « Catullus' Passer as Passer », G\&R, 45, 1998, p. 188-194 ; voir p. 192-193. Mais tous s'accordent sur le caractère érotique des deux poèmes de Catulle.
} 
Frédérique Fleck, «L'épigramme I, 109 de Martial : un poème encomiastique ou satirique ? » Article paru dans la Revue de philologie 82/1, 2008 [2010], p. 49-75.

La suggestion est renforcée par l'apparition du terme osculo au vers suivant ${ }^{34}$. Vont également dans ce sens les termes blandior (3), blando (12), qui évoquent les caresses, et deliciae $^{35}(5)$. Il faut noter aussi l'emploi de collo nixa au vers 8 : cette attitude, qui évoque davantage une personne qu'un animal (on trouve, dans les autres poèmes animaliers, des expressions beaucoup plus nettes à cet égard : in sinu tenere chez Catulle, 2, 2, nec sese a gremio illius mouebat en 3, 8, in sinu iacebat dans CE 1512, 2, molli namque sinu domini dominaeque iacebam dans $\left.C E 1175,7^{36}\right)$, rappelle des passages de poèmes érotiques tels que Properce I, 16, 33 ou III, 4, 15 (nunc iacet alterius felici nixa lacerto ; inque sinu carae nixus ... puellae $\left.{ }^{37}\right)$; l'expression se trouve également dans le poème sur la chienne Patrice $(C E$ 1176), dans un contexte qui renvoie précisément à la poésie érotique : ergo mihi, Patrice, iam non dabis oscula mille / nec poteris collo grata cubare meo. D'autres termes éveillent de semblables échos, bien qu'ils soient employés dans le poème de Martial avec un sens différent : desiderio (10), et suspiria (9). Ces deux termes sont étroitement associés au thème de l'amour (on en voudra pour preuve ce passage des Tusculanes de Cicéron, IV, 72, où l'absence de ces symptômes est présentée comme improbable, voire impossible : [amor] si quis est (...) sine desiderio, sine cura, sine suspirio ${ }^{38}$ ). Desiderio renvoie en outre au vers $5 \mathrm{du}$ poème 2 de Catulle, où le terme se trouve sous la même forme ${ }^{39}$ et à la même place dans le vers, pour désigner métonymiquement Lesbie, objet du désir amoureux du poète. Peut-être est-il possible de lire en filigrane, derrière les vers 8 et 9, capitque somnos / ut suspiria nulla sentiantur, l'expression du contentement que procure un désir assouvi ( $c f$. aussi l'emploi de leuari au v. 13), étant donné que les suspiria sont poussés par l'amant qui, seul et tourmenté, ne parvient pas à trouver le sommeil ${ }^{40}$. Le nom même de la chienne, Issa, semble renvoyer au pronom ipsa dont la prononciation était identique, et qui sert à désigner la femme aimée dans la poésie érotique ${ }^{41}$. Le désir de Publius de posséder un portrait d'Issa fait du reste penser à l'attitude de l'amant qui brûle d'avoir toujours avec lui le portrait de la femme aimée ${ }^{42}$ et l'emploi de toro, au vers 12 , évoque le «lit nuptial »" ${ }^{43}$, bien que le sens de ce terme ait évolué, lui permettant de désigner toute sorte de couche. Le fait que Publius n'arrive pas à trouver de prétendant digne d'Issa ne serait-il pas lié au désir jaloux de la conserver pour lui seul ? C'est ce que semble bien pouvoir suggérer le texte de cette épigramme, avec ce

\footnotetext{
${ }^{34}$ Si la colombe, dont les baisers sont évoqués au vers 2, symbolise dans certains textes la chasteté, elle apparaît aussi ailleurs comme le parangon de la lascivité, notamment chez Catulle, c. 68, 125-128: Nec tantum niueo gauisa est ulla columbo / compar, quae multo dicitur improbius / oscula mordenti semper decerpere rostro / quam quae praecipue multiuola est mulier, « la colombe n'a jamais tant joui de son compagnon au plumage de neige, elle qui, dit-on, le mord sans cesse de son bec, lui arrachant des baisers avec moins de retenue que la femme la plus insatiable », mais aussi chez Martial (XI, 104, 9 et XII, 65, 7-8). M. Citroni, 1975, p. 337, a bien signalé l'ambiguïté de ce passage.

${ }^{35}$ Sur l'emploi euphémistique de deliciae pour désigner les relations extra-conjugales notamment, voir J. N. Adams, 1982, p. 196-197.

36 «Qu'elle tenait sur son sein »; « il ne s'éloignait jamais de son giron»; « elle reposait sur son sein »; « je reposais sur le doux sein de mon maître et de ma maîtresse ».

37 «Elle repose maintenant appuyée sur le bras fortuné d'un autre»; «et, penché sur le sein de ma tendre amie $\gg$.

38 "S'il peut exister une forme d'amour sans désir, sans tourment, sans soupirs ».

${ }^{39}$ Le terme se trouve à l'ablatif singulier chez Martial et au datif singulier chez Catulle, mais ces deux formes sont homonymes.

${ }^{40}$ Voir, entre autres, pour l'évocation des soupirs de l'amoureux qui ne trouve pas le sommeil, Tib. III, 6, 61 ; Prop. II, 22b, 47 ; III, 8, 27 ; Ov., Am. II, 19, 55.

${ }^{41}$ M. Citroni cite notamment à ce propos Ovide, Am. I, 7, 20 ; A. A. II, 171 ; Rem. 304 et 636.

${ }^{42}$ Sur ce sujet, voir l'ouvrage de M. Bettini, Il ritrato dell'amante, Turin, Einaudi, 1992.

${ }^{43}$ Le terme se trouve dans $C E 1175,8$, pour désigner la couche commune du maître et de la maîtresse (mentionnés au vers précédents) où la chienne Margarita était admise.
} 
Frédérique Fleck, «L'épigramme I, 109 de Martial : un poème encomiastique ou satirique ? »

Article paru dans la Revue de philologie 82/1, 2008 [2010], p. 49-75.

faisceau de termes à double résonance ${ }^{44}$. On pourrait en voir une confirmation dans le poème 87 du livre VII où il est dit que Publius « brûle d'amour » pour sa chienne (flagrat amore, 3), expression beaucoup plus forte que celles qui sont employées à propos des autres personnages évoqués (gaudet, fruitur, amat, delectat, placet).

Si l'on considère que Publius est présenté comme un maître complètement épris de sa chienne et que le texte suggère même qu'il pourrait entretenir avec elle des relations peu avouables, on admettra qu'il faut bien qu'une intention satirique ait présidé à la rédaction de ce poème.

\section{Une étrange épigramme}

Les particularités que nous venons de relever concernant la présentation d'Issa et de ses rapports avec son maître ne sont pas les seuls éléments qui nous invitent à proposer une relecture de ce poème ; dans la manière même de cette pièce, plusieurs traits sont de nature à surprendre le lecteur de Martial et appellent, nous semble-t-il, une explication.

Cette épigramme de vingt-trois vers compte parmi les plus longues de Martial, longueur qui est d'ailleurs soulignée par l'épigramme suivante ${ }^{45}$. Or, à cette longueur déjà peu ordinaire s'ajoute le caractère lâche de la composition de cette pièce. Celle-ci apparaît en effet comme une simple juxtaposition de topoï, en contraste avec la structure habituellement très argumentée des épigrammes ${ }^{46}$. Les vers 1 à 5 forment ainsi un premier ensemble accumulant des expressions louangeuses parallèles qui reprennent des formulations consacrées ${ }^{47}$. Le vers 6 développe ensuite le motif conventionnel de l'animal que l'expressivité de ses mimiques et de son jappement rend presque humain; on peut rapprocher ce motif du et plus quam licuit muto canis ore loquebar (« et, avec ma gueule muette de chien, je m'exprimais plus qu'il ne m'était loisible ») de $C E 1175,9$. Il est prolongé par le vers 7 qui pousse plus avant cette idée d'humanité en accordant à la chienne les mêmes sentiments (voire la même pénétration : sentire a les deux sens) qu'aux humains ( $c f$. aussi $C E 1176,7$, déjà mentionné : morib(us) argutis hominem similare paratam). Les deux vers suivants constituent un petit tableau, également convenu, de l'animal se reposant dans les bras de son maître, $c f$. les passages déjà cités in sinu tenere (Catulle, 2, 2), in sinu iacebat $(C E 1512,2)$, molli namque sinu domini dominaeque iacebam (CE 1175, 7), collo grata cubare meo $(C E 1176,4)$; la précision comique ut suspiria nulla sentiantur (elle ne ronfle pas!) semble, elle, originale. Les quatre vers qui évoquent ensuite le comportement de la chienne lorsqu'elle veut faire ses besoins forment avec les précédents un petit ensemble, puisqu'il s'agit toujours du moment du repos

\footnotetext{
${ }^{44}$ Pour une allusion semblable, voir Juvénal 6, 652-654 : Spectant subeuntem fata mariti / Alcestim, et similis si permutatio detur, / morte uiri cupiant animam seruare catellae, « Elles regardent Alceste mourant à la place de son époux et, si la possibilité de faire un tel échange leur était donnée, elles désireraient conserver par la mort de leur mari la vie de leur petite chienne ».

${ }^{45}$ Scribere me quereris, Velox, epigrammata longa. / Ipse nihil scribis : tu breuiora facis, « Tu te plains, Véloce, de ce que j'écris de longues épigrammes. Toi-même, tu n'écris rien : tu fais plus court, assurément. »

${ }^{46}$ Voir la remarque de P. Laurens, 1989, p. 306: «l'épigramme moyenne est caractérisée (...) dans son ensemble par une très forte organisation du développement $»$. Or il semble bien que fassent défaut à cette pièce la cohérence et le resserrement de l'expression, arguments avancés par Martial lui-même pour défendre les épigrammes longues (voir II, 77, 5-7 : ... Marsi doctique Pedonis / saepe duplex unum pagina tractat opus. / Non sunt longa quibus nihil est quod demere possis. « Souvent deux pages de Marsus ou du docte Pedo traitent d'un seul sujet. Elles ne sont pas longues puisque l'on ne peut rien en retrancher. »)

${ }^{47}$ Pour osculo columbae et carior Indicis lapillis, voir les rapprochements indiqués par M. Citroni (1975, p. 337) ; quant à blandior omnibus puellis, l'association de ce substantif et de cet adjectif se trouve notamment chez Ovide, Rem. 717 et Am. II, 2, 34.
} 
Frédérique Fleck, «L'épigramme I, 109 de Martial : un poème encomiastique ou satirique ? » Article paru dans la Revue de philologie 82/1, 2008 [2010], p. 49-75.

et de l'attitude policée d'Issa ${ }^{48}$. Si le fait que l'animal était admis sur la couche de ses maîtres se retrouve régulièrement, aucun parallèle, en revanche, ne semble exister concernant la mention des besoins naturels. La suite et fin du poème est entièrement originale, mais tout aussi décousue : il s'agit de la recherche d'un mari pour Issa aux vers 14 à 16 puis, dans les vers 17 à 23, du portrait que Publius fait faire de sa chienne. En dehors du fait qu'ils se rapportent tous à Issa, ces différents passages n'entretiennent entre eux aucun rapport qui justifierait leur choix et leur ordre de succession.

P. Laurens (1989, p. 309) considère que ce poème présente, pour sa structure, ce qu'il appelle l'ordre embrassé, type de composition repris à Catulle : à l'éloge d'Issa (1-5) répondrait l'éloge de son portrait (17-23), encadrant le développement central, "l'évocation pittoresque de la petite chienne ». Nous sommes pourtant bien loin de la structure annulaire canonique, où le dernier vers reprend à l'identique, ou avec une légère variation, le premier vers du poème (Catulle, 52 ; Martial, IV, 91 ; IV, 64 ; VII, 17) - ce que P. Laurens admet d'une certaine manière puisqu'il parle d'une " application plus souple du procédé » : pas de reprise textuelle ici, et ce sont deux ensembles, de cinq et sept vers respectivement, qui sont censés former la boucle. Cette analyse nous semble en fait avoir été inspirée par l'idée que cette pièce constituerait une variation sur le thème du Moineau de Catulle ${ }^{49}$ et emprunterait ainsi également à cet auteur son mode de composition.

Le style répétitif de ce poème est aussi tout à fait remarquable ${ }^{50}$. Les cinq premiers vers donnent le ton à cet égard, avec l'anaphore de Issa est et la reprise, v. 1-4, d'une même structure syntaxique (comparatif avec complément à l'ablatif). Remarquons que les quatre premiers vers ont par ailleurs un schéma rythmique et accentuel en tout point identique : à passere (trisyllabe, longue suivie de deux brèves) correspondent purior, blandior et carior, puis osculo, omnibus et Indicis se superposent à nequior (nouveau trisyllabe, structure longue - brève - longue) et, enfin, à Catulli (trisyllabe toujours, brève suivie de deux longues) répondent columbae, puellis et lapillis; les accents de mots frappent à chaque fois les première, troisième, sixième et dixième syllabes et la pause principale se situe uniformément après la cinquième syllabe. ${ }^{51} \mathrm{Ce}$ passage a été rapproché ${ }^{52}$ des épigrammes $\mathrm{I}, 39$, où l'expression si quis revient au début de chaque distique, et surtout $\mathrm{V}, 24$, où le nom du gladiateur Hermès, dont le poème fait l'éloge, est répété à l'initiale de chaque vers, à la manière du nom du dieu dans un hymne. Cependant, le procédé n'est, ici, pas tout à fait comparable dans la mesure où l'anaphore ne concerne que 5 vers sur un total de $23:$ le retour d'un même mot ou d'une même expression amène d'habitude jusqu'à la pointe qu'il prépare $^{53}$, au lieu qu'ici la répétition ne concerne qu'un premier mouvement de la pièce ${ }^{54}$.

\footnotetext{
${ }^{48}$ Même enchaînement dans $C E$ 1512, 2-3 : ... in sinu iacebat / somni conscia semper et cubilis, et dans $C E$ 1175, 7-8 : molli namque sinu domini dominaeque iacebam / et noram in strato lassa cubare toro.

${ }^{49} \mathrm{P}$. Laurens conclut son analyse de la structure de cette pièce en remarquant : « Ici encore il s'agit d'une pièce écrite en référence expresse à Catulle ».

50 P. Laurens, «Traduire Martial», RÉL, 76, 1998, p. 200-215, parle, p. 207, de « l'énergie, uis, épigrammatique, fruit de la brièveté, de l'aisance, de la netteté » et estime, à juste titre, que la " première qualité [de l'épigramme] et pour ainsi dire sa forme est la brièveté ».

${ }^{51}$ L'épigramme V, 37, à la mémoire de la petite Erotion, présente en ses treize premiers vers une suite de louanges semblables, mais avec un net souci de uariatio; seuls les trois premiers vers ont une structure syntaxique - et métrique, pour les vers 2 et 3 - parallèle, et ce parallélisme n'est pas redoublé par leur structure rythmique, ni par la présence d'une anaphore. Sur la métrique des épigrammes de Martial, voir R. M. Marina Saez, La Métrica de los epigramas de Marcial. Esquemas rítmicos y esquemas verbales, Zaragoza, Institución «Fernando el Católico », 1998.

52 J. P. Sullivan, 1991, p. 102, note 38 et P. Howell, 1980, p. 334-335.

${ }^{53}$ Voir P. Laurens, 1989, p. 272-284 (« Répétition et structure dialectique de l'épigramme ») et p. $270-272$ (« Le retour du vocatif et la syntaxe de l'ironie »).
} 
Frédérique Fleck, «L'épigramme I, 109 de Martial : un poème encomiastique ou satirique ? » Article paru dans la Revue de philologie 82/1, 2008 [2010], p. 49-75.

Inversement, la répétition semble tout de même trop étendue pour qu'on en rende simplement compte, comme le fait P. Howell (1980, p. 334), par une volonté d'imiter le poème 3 de Catulle (3-4: passer mortuus est meae puellae, / passer, deliciae meae puellae). Il faut souligner également le fait que la répétition sur quatre vers d'un rythme rigoureusement identique est chose tout à fait exceptionnelle chez Martial ${ }^{55}$; c'est donc là un phénomène pour lequel une explication mérite aussi d'être fournie.

Le vers 7, avec le double complément tristitiamque gaudiumque dans lequel chacun des termes est suivi du coordonnant enclitique -que ne se signale pas non plus par sa légèreté et par sa concision. On peut en dire autant de l'expression périphrastique capit somnos du vers suivant, presque redondante par rapport au verbe cubat qui la précède ; redondants aussi les deux verbes monet et rogat du vers 13 et, au vers 16, l'adverbe intensif tam par rapport à l'adjectif tantus du vers 14 .

Mais c'est dans le dernier passage, qui s'étend du vers 17 au vers 23, que les répétitions se multiplient de manière frappante : picta, à l'initiale du vers 18 , se retrouve dans pictam, en position finale au vers 23 , tabella se trouve à la fin des vers 18 et 21 , l'expression tam similem du vers 19 est répétée par tam similis qui occupe la même place dans le vers suivant, les deux derniers vers enfin sont identiques au dernier mot près, ueram en 22 et pictam (qui constitue une répétition par rapport au v. 18) en 23, et ils reprennent le putabis déjà présent au vers 6 . Ce manque de resserrement est en rupture avec l'esthétique de la pointe $^{56}$, et il semble d'ailleurs difficile de trouver dans ce poème quelque chose qui ressemble à une pointe, cet élément pourtant essentiel de l'épigramme martialienne. L'idée selon laquelle les deux derniers vers formeraient un concetto portant sur la ressemblance de l'œuvre d'art avec la réalité ${ }^{57}$ ne nous semble guère fondée. C'est là un topos qui ne pourrait fournir le trait que s'il était travaillé à cette fin, comme en III, 35 (Artis Phidiacae toreuma clarum / pisces aspicis : adde aquam, natabunt, " Tu vois ces poissons, ce célèbre relief ciselé, œuvre de Phidias : mets-y de l'eau et ils nageront »), où la pointe que constituent les trois derniers mots repose sur une exagération (natabunt) - absente dans notre poème à cause de la modalisation liée à la présence de putabis - et sur l'introduction d'une condition fictive qui fonde l'exagération (adde aquam) ${ }^{58}$. Par ailleurs, du point de vue du sens, utramque putabis esse ueram pourrait constituer une conclusion acceptable, mais que penser de la véritable formule conclusive, utramque putabis esse pictam, au regard des conceptions antiques sur l'art ? Étrange accomplissement que celui qui consisterait à faire paraître peint le modèle. Rien de piquant donc à ce qu'il semble dans cette fin, pas de jeu sur les mots ${ }^{59}$ l'opposition des adjectifs ueram et pictam est bien mise en valeur par la reprise textuelle des autres éléments des deux derniers vers, mais quel sens peut-on lui donner dans le cadre d'une lecture encomiastique ? -, nulle concision. On serait presque tenté, tant ils semblent répétitifs et dépourvus de relief, de considérer les derniers vers comme une sorte d'anti-pointe.

L'aspect décousu de cette pièce, son caractère répétitif encore accentué par sa longueur et, plus particulièrement, l'anaphore employée dans les premiers vers seulement

\footnotetext{
${ }^{54}$ On verra plus loin que le procédé est semblable dans son principe ; mais seule une lecture sensible à l'ironie permet d'en rendre compte.

${ }^{55}$ C'est ce que souligne J. P. Sullivan, 1991, p. 229.

${ }^{56}$ P. Laurens, 1998, p. 207, rappelle ainsi que la brièveté, « bienvenue dans tout le corps de l'épigramme », est " exigible au premier chef dans le trait final, la flèche ».

${ }^{57}$ M. Citroni, 1975, p. $340:$ : il concetto che la somiglianza è tale che non si può distinguere tra il soggetto e il suo ritratto ».

${ }^{58}$ Le fonctionnement de la pointe dans cette épigramme III, 35 a été analysé par P. Laurens, 1989, p. 366-367.

${ }^{59}$ Selon P. Laurens, 1989, p. 370, la « pointe verbale » est « la féconde origine de toutes les autres, car, si l'on y prend garde, toutes s'aident des mots et de leurs significations $»$.
} 
Frédérique Fleck, «L'épigramme I, 109 de Martial : un poème encomiastique ou satirique ? »

Article paru dans la Revue de philologie 82/1, 2008 [2010], p. 49-75.

ainsi que la clôture pesante de l'épigramme constituent autant de traits exceptionnels chez Martial dont il nous parait indispensable de rendre compte.

\section{Proposition de lecture polyphonique}

\subsection{Une situation d'énonciation ambiguë}

Nous avons déjà remarqué que le poème I, 109 de Martial se distingue, d'un point de vue énonciatif, des autres textes, littéraires ou épigraphiques, consacrés à des animaux familiers par l'absence d'adresse à l'animal ou à son maître. Il en diffère encore, de manière plus remarquable, par l'absence de marque de première personne du singulier, qui devrait conduire à s'interroger sur l'attribution des vingt-trois vers du poème. Dès lors que l'on se pose cette question - trop souvent négligée du fait qu'il paraît acquis d'emblée que c'est le poète qui s'exprime - on s'aperçoit que les marques de personne qui permettraient de clarifier la situation d'énonciation sont absentes ou ambiguës.

Ainsi, au vers 15 , se présente une forme verbale à la première personne du pluriel, inuenimus, mais il est difficile d'en tirer une quelconque conclusion, étant donné que trois interprétations en sont possibles : cela peut se traduire par «nous trouvons », «je trouve » («nous » d'auteur courant dans la poésie latine) ou encore « on trouve ». Pour ce qui est de Publius, son nom apparaît bien à deux reprises, aux vers 5 et 18, mais, dans le reste du texte, il semble que les termes qui auraient dû le désigner aient été soigneusement effacés. Cet effacement volontaire apparaît nettement dans les vers 8 à 13, où il est question du comportement d'Issa avec son maître. Alors que, dans les passages équivalents des poèmes de Catulle, les termes renvoyant à Lesbie sont présents presque à chaque vers, Publius est notoirement absent de cette évocation. On relève ainsi au vers 8 l'expression collo nixa, dans laquelle aucun terme ne vient indiquer la personne - Publius, évidemment - sur le cou de laquelle la chienne s'appuie ${ }^{60}$. De même, on pourrait attendre une mention de Publius au vers précédent, si l'on comprend, comme c'est vraisemblable, que c'est la peine et la joie de son maître que la chienne est capable de ressentir. Au vers 9, c'est une tournure passive qui a été choisie, suspiria nulla sentiantur, ce qui permet de ne pas mentionner la personne, toujours Publius, qui est le siège de la perception. L'absence de complément du verbe suscitat au vers 12 , puis des verbes monet et rogat au vers 13 est encore plus remarquable. ${ }^{61}$

\footnotetext{
${ }^{60}$ Cette imprécision a dérouté E. Courtney, Musa Lapidaria. A Selection of Latin Verse Inscriptions, Atlanta, Scholars Press, 1995, p. 409 qui, dans son commentaire de $C E 1176$ sur la chienne Patrice, relève la différence entre le v. 4 de ce poème, collo ... cubare meo, et le v. 8 de l'épigramme de Martial et en déduit que collo nixa cubat « means that the dog sleeps curled up so that it seems to be lying on its own neck». La traduction italienne de G. Norcio, Epigrammi di Marco Valerio Marziale, Torino, UTET, 1980, témoigne d'une interprétation semblable : «con la testa reclinata sta buona ».

${ }^{61}$ M. Citroni, 1975, p. 338-339, remarque ces emplois absolus sans s'interroger sur leur raison d'être. Certains traducteurs n'ont pas été arrêtés par cette absence de marques. Ainsi J. M. C. Toynbee, 1973, p. 122, qui traduit en suppléant allègrement les indices de personne manquant: "Sorrow and joy she feels as much as he does, I snuggling close to his neck she sleeps so softly / that you'd scarcely believe the pet was briething. / If in the night she finds that Nature's calling, / Never a spot she'd leave on master's bedspread, / But with her paw a gentle tap she gives him, / 'Please put me down' - and then, 'Please pick me up now'». Même chose dans la collection Loeb, traduction de D. R. Shackleton Bailey, 1993 : "She feels both his sadness and his joy. She lies resting on his neck and slumbers, with not a breath perceptible. Urged by her belly's need, she never betrays the bedcover by a single drop, but rouses him with a wheedling paw and warns to put her down from the bed and then asks to be taken up. », ou encore dans l'édition commentée du livre I par P. Howell, 1980 : "She leans up against his neck and sleeps, so quietly that he never feels her sighing, and when overcome by the needs of her bladder she has never sullied the bed-clothes with a single drop, but instead she wakes him up with coaxing paw, and warns
} 
Frédérique Fleck, «L'épigramme I, 109 de Martial : un poème encomiastique ou satirique ? » Article paru dans la Revue de philologie 82/1, 2008 [2010], p. 49-75.

Pour discrets qu'ils soient, les indices concernant la situation d'énonciation ne sont pourtant pas complètement absents du texte. En effet, si la première personne du singulier n'apparaît pas, la deuxième personne du singulier est, elle, bien présente : tu ... putabis (6), uidebis (19), pone (21) et putabis (22 et 23). Cette deuxième personne du singulier semble généralement comprise comme une expression de l'indéfini ${ }^{62}$. Or une telle lecture n'est grammaticalement pas possible. Si l'emploi de la deuxième personne du singulier au subjonctif constitue une des manières les plus courantes d'exprimer l'indéfini en latin, il n'en va pas de même de son emploi à l'indicatif futur et à l'impératif présent; de plus, l'emploi du pronom personnel est alors évité, ce qui n'est pas le cas dans notre texte $(t u, 6)$. Ces formes de deuxième personne du singulier doivent se rapporter à un interlocuteur déterminé. Celui-ci est même interpellé au vers 6 , avec $t u$ (en latin, le pronom personnel n'apparaît au nominatif que dans le cadre d'une antithèse, quand il est suivi d'une apposition ou pour procéder à une interpellation; les deux premiers cas sont ici exclus), et pris directement à partie au vers 21 avec l'impératif pone. Mais qui est-il exactement? Aucun terme d'adresse ne le désigne, chose rare dans les épigrammes ${ }^{63}$, ce qui produit là encore un effet d'indétermination. Il pourrait s'agir d'une adresse au lecteur-spectateur telle qu'on en trouve dans certaines épigrammes décrivant des œuvres d'art; ce procédé repose sur le modèle des épigrammes gravées, par exemple, sur le socle d'une statue, qui instaurent un dialogue fictif avec le passant. Si cette lecture s'accorde avec la fin de notre poème, elle convient cependant moins bien à son début, qui n'a rien d'une ekphrasis.

À qui peut-on identifier alors cet interlocuteur, et qui est le locuteur ? Si aucune forme, dans le texte, ne relève proprement de la première personne du singulier, il faut toutefois noter l'emploi, au début des vers 6 et 17, de la forme pronominale hanc, désignant Issa. On s'attendrait en effet à trouver, pour désigner la chienne, l'anaphorique eam ou le pronom à valeur laudative illam. L'emploi de hanc pourrait être analysé comme déictique ce qui supposerait que l'épigramme est écrite tout entière sur le modèle des textes accompagnant des œuvres d'art, la chienne Issa étant désignée comme le chef-d'œuvre offert à l'admiration -, mais il est possible aussi de le comprendre en donnant au pronom sa valeur personnelle, qui renvoie à la sphère du locuteur (ce qui est proche de lui, ce qui lui appartient). C'est dans la bouche de Publius, le maitre d'Issa, que ce pronom aurait alors sa place. On est de la sorte tenté d'attribuer à Publius ces énoncés, dont le destinataire, désigné par les formes de deuxième personne, ne serait autre que Martial (en tant que figure d'auteur); la forme de première personne du pluriel (inuenimus) du vers 15 représenterait alors un "nous » de majesté renvoyant au seul Publius, à moins de supposer que celui-ci ait réussi à associer quelques amis à sa recherche.

him to put her down from the couch, and then she asks to be picked up again. » Plus discrètement, chez P. Barié et W. Schindler, 1999: «An seinen Hals gelehnt, ruht sie und schläft ein, / ohne daß man dabei ihren Atem spürt».

${ }^{62}$ Ainsi dans les traductions par « vous » en français et, peut-être aussi, dans les traductions anglaises où « you » est ambigu. C'est très nettement le cas dans la traduction allemande de P. Barié et W. Schindler, 1999, où le putabis du vers 6 est rendu par «meint man» et uidebis, 19, par «man ... feststellen kann»; de manière significative, l'impératif pone du vers 21 force les traducteurs à passer à la deuxième personne du singulier (« setze »), qui est ensuite conservée pour rendre les putabis des deux vers suivants. De manière semblable, H.-J. Izaac, 1930-1933, passe de « vous croiriez » pour la traduction du vers 6 à « tu verras », « mets » et « tu croiras » pour la fin du poème. Les adaptations anciennes citées par E.-T. Simon, 1819, vol. 3, p. 384 et 392-394, conservent le «vous » jusqu'à la fin (Pommereul, Eloi Johanneau), quand elles n'emploient pas « on» (T. S. Des Accords : « de toi-même l'on diroit / que ta pourtraiture on voit », 63-64).

${ }^{63}$ Sur la quasi-nécessité de l'emploi de termes d'adresse en latin, voir E. Dickey, Latin Forms of Address from Plautus to Apuleius, Oxford, Oxford University Press, 2002, p. 252-255. 
Frédérique Fleck, «L'épigramme I, 109 de Martial : un poème encomiastique ou satirique ? » Article paru dans la Revue de philologie 82/1, 2008 [2010], p. 49-75.

Si l'on admet cette hypothèse, l'épigramme que nous étudions présente, du point de vue de l'énonciation, une structure complexe, car les voix du sujet lyrique et de Publius s'y mêlent. Les quatre premiers vers constituent quatre énoncés rapportés au style direct libre, dont l'énonciateur est Publius ${ }^{64}$; le vers 5 , où Publius est mentionné, est un contrepoint ironique de l'auteur. Les vers 6 à 16 forment une nouvelle séquence de discours rapporté au style direct libre, attribuable également à Publius. L'énonciateur des vers 17 à 21 est Martial, puisque Publius apparaît à la troisième personne au vers 18; mais l'auteur ne prend réellement en charge que le contenu, très neutre, de la proposition principale, c'est-à-dire du seul vers 18. Dans la proposition subordonnée de but qui précède et dans la proposition relative qui suit, ce sont les propos d'un autre énonciateur, Publius, qu'il nous fait à nouveau entendre, sous la forme, cette fois, de mentions ; ces propos ne sont pas assumés par Martial, qui s'en distancie. Enfin, les vers 21 à 23 constituent un dernier passage de discours rapporté au style direct libre attribuable au maître d'Issa. Avant de développer cette analyse, arrêtonsnous sur quelques points qui méritent d'être précisés.

Cette interprétation met en évidence la présence de plusieurs passages de discours rapporté au style direct libre ${ }^{65}$. Il s'agit d'un type de discours rapporté qui n'est pas transposé (un discours direct, donc), et qui est « libre » du fait de son autonomie syntaxique (il n'est pas régi par un verbe introducteur) ${ }^{66}$. La présence d'un passage de discours rapporté au style direct libre est signalée contextuellement par une rupture énonciative (qui peut concerner les temps, les personnes, la deixis), mais ces indications sont plus ou moins nettes, et il arrive que l'identification de niveaux d'énonciation différents ne puisse se faire que grâce à des données extralinguistiques (par exemple grâce à la connaissance des opinions du locuteur, qui sont en rupture avec les opinions exprimées dans le passage de discours rapporté). Ainsi, dans notre texte, la rupture énonciative est difficile à repérer. Cela tient, pour une part, à la quasi-absence du discours citant qui sert normalement de point de repère (il n'occupe en tout et pour tout que deux vers) ainsi qu'à son apparition tardive, puisque l'épigramme nous présente d'abord quatre vers de discours cité et qu'il faut attendre le cinquième vers pour trouver le cadre permettant d'interpréter le passage qui précède en rapportant son énonciation à Publius. D'autre part, l'interprétation des marques du changement de plan énonciatif est délicate, quand ces marques ne sont pas tout simplement absentes : il n'y a pas de rupture temporelle étant donné que le discours citant est lui-même au présent et, comme nous l'avons déjà observé, la variation des marques de personne n'est guère apparente.

Il faut en effet souligner - c'est un point qui a son importance - que le texte ne se livre pas immédiatement, car son sens se dérobe sous une ambiguïté soigneusement ménagée ${ }^{67}$. Il joue en particulier des équivoques constitutives du discours au style direct libre et de la

\footnotetext{
${ }^{64}$ Signalons que l'adaptation de Pommereul, citée par E.-T. Simon, 1819, vol. 3, p. 384, dans laquelle le sujet lyrique prend explicitement en charge ces louanges des premiers vers, ne permet plus cette interprétation: « nulle bête, à mon gré, n'a les yeux si brillans » (v. 3).

${ }^{65}$ Les travaux de J. Bayet, « Le style indirect libre en latin », RPh, 57, 1931, p. 327-342 et 58, 1932, p. 5-23, ont montré que le style indirect libre n'était pas une invention des auteurs modernes; le style direct libre, qui lui a succédé comme marqueur linguistique de la modernité, notamment dans la mouvance du nouveau roman (pour ce qui concerne le rapport entre discours direct libre et monologue intérieur, voir L. Rosier, Le Discours rapporté. Histoire, théories, pratiques, Paris-Bruxelles, Duculot, 1999, p. 271-278), a aussi son antiquité, et sans doute une recension des passages de ce type chez les auteurs latins présenterait-elle quelque intérêt.

${ }^{66}$ Cette liberté du discours direct libre est souvent présentée aussi comme résultant de l'absence de tout marquage typographique à l'écrit (deux-points, virgule, guillemets, italiques); ce critère n'est évidemment pas valable pour les textes latins qui n'utilisaient aucun de ces signes de ponctuation.

${ }^{67}$ En témoignent les commentaires et traductions que nous avons eu l'occasion de citer à plusieurs reprises.
} 
mention $^{68}:$ les propos rapportés auraient pu être introduits clairement (« Publius dit à qui veut l'entendre:», " comme le dit Publius »), mais tel n'est pas le cas. De même, comme nous l'avons déjà noté, le texte semble bien avoir été travaillé de sorte que les marques de personne n'apparaissent que le plus rarement possible et soient, alors, discrètes ou ambiguës. Tout le sel de cette épigramme repose ainsi dans la subtile connivence établie avec le lecteur : celui-ci sera-t-il suffisamment sensible aux nuances pour percevoir les dissonances thématiques et formelles $^{69}$ qui lui permettront d'entendre la polyphonique porteuse d'ironie ${ }^{70}$ ? Ces ambiguïtés sources d'une complicité avec les happy few sont courantes chez Martial, mais elles concernent généralement un segment plus limité du texte - mot ou expression -, par exemple dans le cadre d'une syllepse de sens. Le procédé est ici étendu à l'échelle des propositions et même de suites de plusieurs phrases dont l'attribution n'est pas immédiatement évidente, et finalement à l'échelle du poème tout entier, car c'est son interprétation globale qui est en jeu : éloge complaisant ou satire légère ${ }^{71}$.

\subsection{Un pastiche satirique}

Revenons maintenant plus en détail sur l'analyse qu'autorise une lecture polyphonique de l'épigramme. Les vers 1 à 4 reproduisent les propos louangeurs de Publius à l'égard de sa chienne. Le caractère dithyrambique de ces éloges s'explique aisément si c'est au maître d'Issa qu'il faut les attribuer. L'anaphore dépeint alors de façon parodique le travers de Publius qui n'a que le nom d'Issa à la bouche et, tel un amoureux, ne sait parler que d'elle à ses amis (ce procédé est employé pour le nom de Naevia, répété sept fois dans l'épigramme I, 68, satire de l'amant pour qui tout ne tourne qu'autour de celle qu'il aime). La reprise systématique de la même structure et du même schéma rythmique dans ce passage où

\footnotetext{
${ }^{68}$ Le discours au style direct libre et la mention autonymique - quand elle n'est pas signalée, à l'oral par l'intonation, à l'écrit par des guillemets ou des italiques, mais constitue une citation cachée - partagent avec le discours au style indirect libre la caractéristique d'être ambigus quant à leur interprétation, contrairement au discours rapporté direct ou indirect. Ces formes de discours rapporté ne sont pas marquées et relèvent entièrement de l'interprétation contextuelle.

${ }^{69}$ Comme le signale J. Authier-Revuz, « Repères dans le champ du discours rapporté », IG, 55, 1992, p. 38-42 et 56,1993 , p. 10-15, lorsqu'un texte présente des formes de discours rapporté non marquées, "certains indices vont, de façon très claire ou de façon tout à fait incertaine, conduire à interpréter, dans une suite de phrases ou une suite d'éléments, une phrase, ou un élément, comme relevant d'un autre acte d'énonciation » (p. 14). Cet auteur faisait déjà remarquer, dans un article plus ancien (J. Authier, "Les formes du discours rapporté. Remarques syntaxiques et sémantiques à partir des traitements proposés », Documentation et recherche en linguistique allemande contemporaine, Vincennes, 17, 1978, p. 2-87), p. 71-72: «Le décodage par le récepteur $(1=$ il y a un "comme dit x", 2 = "x" est identifiable) repose alors sur la reconnaissance par lui des mots d'un autre. (...) Le risque d'échec de la communication est comme une composante de ce mode de parole. (...) [Ce] risque est ce qui fait le plaisir de certains textes, où le locuteur et le récepteur jouent, sans signal (sans filet, pourrait-on dire), de ces mots du locuteur qui sont les mots d'un autre. Il peut être (...) cultivé comme un jeu par des locuteurs amateurs d'ironie froide. ».

${ }^{70}$ L'approche polyphonique de l'ironie, définie comme l'attribution à un autre énonciateur d'un énoncé par rapport auquel le locuteur prend ses distances, a été inaugurée par D. Sperber et D. Wilson, « Les ironies comme mentions », Poétique, 36, 1978, p. 399-412. La distanciation souligne le manque de justesse ou de pertinence des propos mentionnés. La personne aux énoncés, réels ou imaginaires, ou aux pensées de laquelle il est fait écho est la cible de l'ironie.

${ }^{71}$ É. Wolff, article à paraître, a récemment mis en lumière l'ambiguïté de certains éloges adressés à l'empereur, notamment dans la pièce $79 \mathrm{du}$ livre IX. Concernant les poèmes adressés aux patrons, N. Holzberg, 2002, p. 75, a proposé de voir dans l'épigramme I, 7, où il est question de l'imitation du Moineau de Catulle par Stella, une allusion obscène par laquelle Martial se moque gentiment de son protecteur (la comparaison entre la valeur littéraire respective de la colombe et du moineau pourrait être ramenée à une comparaison entre la taille du membre viril de chacun des auteurs).
} 
Frédérique Fleck, «L'épigramme I, 109 de Martial : un poème encomiastique ou satirique ? »

Article paru dans la Revue de philologie 82/1, 2008 [2010], p. 49-75.

s'accumulent les éloges fait partager au lecteur la lassitude que peuvent ressentir les amis et familiers contraints d'écouter ces discours. Les répétitions et redondances que nous avons relevées par ailleurs tout au long de la pièce doivent recevoir la même interprétation : elles concourent de la même manière à créer une impression de ressassement.

Le vers 5, énoncé pris en charge par l'auteur et qui clôt cette séquence, est un contrepoint ironique. Il s'agit là d'un procédé très courant chez Martial. Souvent, en effet, l'épigrammatiste joue de la répétition d'un terme qu'il charge, lors de sa dernière reprise, d'un sens différent ou bien qu'il éclaire par une nouvelle définition ${ }^{72}$. Or c'est bien une redéfinition d'Issa que nous lisons ici. Alors que les quatre premiers vers la présentent comme la huitième merveille du monde et laissent indécise son identité (on a tout lieu de croire, à ce stade, qu'il s'agit d'une jeune femme), le vers 5 la ramène à ses véritables proportions : ce n'est qu'une chienne $^{73}$, et même une toute petite chienne, comme l'indique le diminutif qui accentue encore, de façon comique, le contraste (il ne s'agit pas, ici, d'un diminutif hypocoristique, mais c'est bel et bien la taille de l'animal qui est évoquée, comme le confirme le vers 3 de l'épigramme VII, 87, Publius exiguae si flagrat amore catellae, « si Publius brûle d'amour pour une minuscule petite chienne »). La précision deliciae Publi permet d'expliquer les éloges qui précèdent et de les rapporter à leur énonciateur, Publius. L'arrivée du contrepoint est marquée, comme c'est habituellement le cas chez Martial, par un changement dans la structure du vers : il s'agit ici, d'une part, de l'abandon du schéma syntaxique avec comparatif accompagné d'un comparant à l'ablatif et, d'autre part, de la rupture avec la cadence des vers précédents (la pause principale, qui se trouvait, dans les vers 1 à 4 , après la cinquième syllabe, est déplacée après la sixième syllabe et les accents de mot frappent les première, quatrième, huitième et dixième syllabes et non plus les première, troisième, sixième et dixième $)^{74}$. En soulignant le manque de justesse de la description qui précède (Issa n'est pas la huitième merveille du monde, Issa n'est pas une jeune femme) et en permettant de l'attribuer à un autre énonciateur, ce cinquième vers révèle le caractère ironique du début de l'épigramme et indique la cible de cette ironie, Publius. Le lecteur est dès lors prévenu et il pourra percevoir les sous-entendus ironiques dans la suite de la pièce : « un animal ne parle pas et n'a pas de sentiments humains » (6 et 7), "Issa n'est pas chaste » (14-15, avec mise en relief de castae en début de vers, alors que le passage qui précède immédiatement suggère une relation érotique entre la chienne et son maitre), «le portrait qui est fait d'Issa n'est pas ressemblant ${ }^{75}(19-23)$.

Le caractère décousu du poème s'explique parfaitement si l'on comprend que celui-ci, au lieu d'être une énonciation attribuable au sujet lyrique ou, comme cela se produit souvent aussi dans les épigrammes de Martial, un bref dialogue fait d'un échange de questions et de

\footnotetext{
${ }^{72}$ C'est le procédé de l'antanaclase. Voir, pour des exemples, P. Laurens, 1989, p. 276-279, « Retour du mot et travail critique », à propos notamment de I, 9 où bellus est repris pour être défini comme équivalant à pusillus, ce qui rend sa combinaison avec magnus impossible, ou de II, 3 où le sens de debere - il ne peut y avoir dette que si l'on est solvable - est dégagé en fin d'épigramme. On peut songer aussi aux précisions apportées parfois par ces reprises, $c f$. le dernier vers de IV, 81 : negare iussi, pernegare non iussi, « je t'ai invitée à refuser, non à persister dans ton refus ", cité par É. Wolff, Martial ou l'apogée de l'épigramme, Rennes, Presses Universitaires de Rennes, 2008, p. 91.

${ }^{73}$ L'effet de surprise est ménagé même à l'intérieur de ce cinquième vers, où le mot catella n'arrive que tardivement.

${ }^{74}$ On trouve couramment, pour souligner la pointe dans les épigrammes, des procédés tels que le déplacement de la coupe, d'un mot ou groupe de mots précédemment répété toujours à la même place dans le vers, ou encore l'introduction d'une apostrophe au vocatif. Voir P. Laurens, 1989, p. 268-272 et passim (p. 329: «c'est la rupture - au moins partielle - du parallélisme qui annonce la chute de l'épigramme »; p. 333 : « la recherche de la concinnitas est brusquement rompue par un effet de uariatio qui n'est point gratuit, mais (...) signale le point culminant et le terme du mouvement rhétorique »).

${ }^{75}$ Nous reviendrons sur ce dernier sous-entendu.
} 
Frédérique Fleck, «L'épigramme I, 109 de Martial : un poème encomiastique ou satirique ? »

Article paru dans la Revue de philologie 82/1, 2008 [2010], p. 49-75.

réponses entre le sujet lyrique et un interlocuteur, ne représente en fait qu'un assemblage de propos rapportés. La longue séquence de discours direct libre qui s'étend du vers 6 au vers 16 ne doit ainsi pas se lire comme une série d'énoncés qui auraient été produits consécutivement, mais plutôt comme un assemblage de trois «morceaux choisis" particulièrement représentatifs. Il en va de même des quatre premiers vers qu'il faut manifestement lire comme quatre fragments de discours autonomes mis par l'auteur à la suite les uns des autres plutôt que comme l'évocation d'un unique acte d'énonciation dans lequel les quatre propositions se feraient suite. De la simple juxtaposition de ces paroles qui ne sont reliées que par leur thème - encore et toujours Issa - se dégage avec plus de force que d'une description construite le portrait d'un maître que n'intéresse que ce qui se rapporte à son animal favori. Notre interprétation conduit par ailleurs à considérer la relative longueur de l'épigramme comme mimétique de la longueur même des discours de Publius qui ne tarit plus quand il s'agit de parler de sa chienne ${ }^{76}$. Les imperfections relevées précédemment dans la facture et la composition du poème, le manque d'originalité du discours sur Issa, essentiellement fait de topoï, apparaissent ainsi in fine comme le comble de l'art, si c'est, comme nous le suggérons, à un pastiche que se livre Martial.

Les dissonances signalées plus haut trouvent elles aussi leur résolution dans le cadre de cette lecture polyphonique; bien plus, elles constituent autant d'indices signalant au lecteur qu'il faut attribuer à un autre qu'à l'auteur la plupart des énoncés du poème ${ }^{77}$. La naïveté du propos, l'enthousiasme débordant, la complaisance pour des détails même scabreux sont à mettre au compte de Publius, et non pas de Martial; et c'est à Publius, non à Martial naturellement, qu'il faut attribuer les préoccupations matrimoniales dont font état les vers 15 et 16. C'est à Publius encore, pastiché par Martial, que l'on doit rapporter l'emploi d'un vocabulaire qui est en décalage avec son objet ${ }^{78}$. Cet usage de termes et d'expressions appartenant à un style élevé, qui convient mal à une conversation amicale, même s'il s'y exprime le naïf désir d'exalter les vertus de l'animal aimé, invite à formuler une hypothèse supplémentaire : les fragments de discours de Publius pourraient relever non pas - ou pas seulement - du langage ordinaire, mais d'un registre poétique. Certains commentateurs ont, en effet, interprété la mention du moineau de Catulle, au vers 1, comme indiquant la volonté de Martial de rivaliser, dans cette pièce, avec le poète de Vérone ${ }^{79}$; or, s'il faut attribuer en fait le contenu de ces vers à Publius, il faut supposer que c'est lui qui aurait essayé de composer, en l'honneur de sa chienne, un poème d'amateur à la façon de Catulle. Ce pourrait être alors le pastiche d'un tel poème que Martial nous donnerait à lire par bribes. La vogue que connaissaient manifestement les poèmes à la manière du Moineau de Catulle et dont Martial lui-même se fait par ailleurs l'écho (dans les épigrammes I, 7 et VII, 14 où il évoque

\footnotetext{
${ }^{76}$ On pourrait y voir un écho dans le poème 198 des Apophoreta: Delicias paruae si uis audire catellae, / narranti breuis est pagina tota mihi, « Si tu veux connaître les délices de cette toute petite chienne, c'est trop peu pour moi d'une page entière pour te les raconter». Les Apophoreta ont été publiés un peu avant le premier livre des Épigrammes, mais Martial pouvait déjà penser au cas de Publius ou même avoir déjà commencé à écrire sa pièce sur Issa.

${ }^{77}$ Le discours au style direct libre et la mention autonymique (lorsqu'elle constitue, comme c'est le cas dans notre texte, une citation cachée) sont, selon les mots de J. Authier-Revuz, 1993, p. 14, "des formes non marquées, purement interprétatives de représentation d'un autre acte d'énonciation »; les indices permettant de les interpréter comme telles sont notamment l'absence de cohérence logique entre les différents énoncés d'un texte et l'absence d'homogénéité des « manières de dire ». Ainsi, par exemple, l'expression lux suprema du vers 17, qui relève du style noble - épique ou tragique -, est un signal textuel qui invite à attribuer à Publius plutôt qu'à Martial le contenu de la subordonnée.

${ }^{78}$ Le pastiche satirique, rebaptisé « charge » par G. Genette, Palimpsestes. La littérature au second degré, Paris, Seuil, 1982, p. 40, se caractérise par une manière de s'exprimer rendue ridicule «par un procédé d'exagérations et de grossissements stylistiques » (p. 39) ; le pastiche héroï-comique constitue une variété de la charge.

${ }^{79}$ Ainsi P. Richard, 1931.
} 
Frédérique Fleck, «L'épigramme I, 109 de Martial : un poème encomiastique ou satirique ? »

Article paru dans la Revue de philologie 82/1, 2008 [2010], p. 49-75.

une composition de son ami Stella) constitue un argument en faveur de cette hypothèse. Précisons toutefois que, si le Publius dont il est question est probablement une personne réelle, connue de Martial, il n'est en revanche pas nécessaire qu'il ait véritablement écrit un tel poème ou tenu les propos que lui prête Martial ${ }^{80}$. On considérera plutôt que le poème de Publius sur sa chienne Issa est un hypotexte fictif ${ }^{81}$ que suppose et construit l'épigramme, tout comme ce serait le cas s'il s'agissait d'un personnage fictif : les propos rapportés et le poème supposé ne sont vraisemblablement qu'invention de l'auteur.

Dans ces bribes de paroles ou de poème qu'il prête à Publius, Martial glisse des termes qui, nous l'avons vu, suggèrent une relation érotique entre le maître et sa chienne. On peut parler à ce propos d'ironie d'auteur puisque ces allusions érotiques à visée satirique ne sauraient être attribuées au Publius mis en scène dans l'épigramme. Celui-ci, naturellement, ne désire exprimer qu'une naïve affection. $\mathrm{Si}$, dans ces conditions, l'on voulait supposer que le poème de Martial s'appuie sur des paroles réellement prononcées par ledit Publius, il ne serait pas suffisant de considérer qu'il s'agit simplement de citations tronquées. Car ni une sélection habile, ni un montage savant des paroles de ce dernier ne pourraient suffire à produire un contrepoint aussi nettement ironique. Si l'on suppose donc que Martial reprend les propos de ce Publius, il faut admettre qu'il ne le fait que dans le cadre de ce que J. Authier nomme le discours direct «pseudo-textuel ${ }^{82}$, c'est-à-dire d'une reformulation. En l'occurrence, la reformulation aurait abouti à substituer un vocabulaire aux connotations nettement érotiques à des termes qui devaient n'exprimer que la tendresse et l'affection. Cependant, répétons-le, il n'est nullement nécessaire que Publius ait réellement tenu des propos semblables à ceux que Martial lui prête : le discours qui lui est attribué dans le poème peut fort bien n'être que pure invention.

Il nous faut, enfin, essayer de rendre compte des sept derniers vers et, plus spécifiquement, de ce que nous avons appelé l'anti-pointe de ce poème. Pourquoi cette fin apparemment si éloignée de ce que l'on trouve habituellement chez Martial ? Les répétitions, on l'a vu, sont un procédé qui apparaît dans l'ensemble du poème, mais cela ne suffit pas à expliquer l'usage étendu qui en est fait à l'endroit où elles semblent le plus malencontreuses. Pourquoi, aussi, avoir introduit ce thème de la peinture, que l'on ne rencontre dans aucun des autres textes se rapportant à des animaux familiers, et auquel sa position finale et le nombre de vers qui lui est accordé confèrent une importance toute particulière ? Il est naturellement tentant de considérer que l'introduction du thème du portrait constitue une mise en abyme, le portrait peint étant une figure de cette autre œuvre d'art qu'est le portrait littéraire de l'animal - le parallèle entre art pictural et art littéraire est explicite dans l'épigramme VII, 84, où Martial compare, pour la ressemblance, le portrait peint qui est fait de lui à celui qui se dégage de son recueil de poèmes - mais cette supposition n'a, par elle-même, qu'une valeur explicative très limitée. Quel est, enfin, le sens du dernier vers ? On comprend mal, nous l'avons déjà signalé, l'application à Issa de l'adjectif pictam : cette dernière affirmation paraît incongrue dans le cadre de l'éloge d'une œuvre artistique (si le comble de l'art est de peindre un portrait qui ait l'air vivant, comme l'admettaient les Anciens, le poème devrait s'achever sur l'avant-dernier vers), et on ne voit pas non plus en quoi elle servirait l'éloge de la chienne. Serait-ce que Publius, dans son désir de souligner la ressemblance extrême entre le tableau et son modèle, se serait laissé entraîner trop loin par les jeux de miroir?

\footnotetext{
${ }^{80} \mathrm{Si}$ tel était toutefois le cas, nous gagnerions une indication biographique concernant Publius : celui-ci aurait été poète à ses heures.

${ }^{81}$ Hypotexte qui serait lui-même un hypertexte par rapport au poème de Catulle.

${ }^{82} \mathrm{~J}$. Authier, 1978, p. 75, appelle ainsi les cas, très fréquents, où le locuteur principal, au lieu de répéter les mots du locuteur de l'acte d'énonciation rapporté, «propose, au discours direct, comme rapport de l'acte de parole rapporté, (...) ses propres mots à lui ».
} 
Le fait que la fin de l'épigramme prenne si nettement le contre-pied de l'esthétique de la pointe caractéristique de l'écriture de Martial constitue, à notre avis, un dernier signal de discordance, invitant le lecteur à s'interroger sur l'énonciation de la pièce. Ce qui est dit du tableau est une indication supplémentaire : l'insistance sur l'extrême ressemblance du portrait dénonce l'existence d'un locuteur enthousiaste qui a toute chance d'être autre que le sujet lyrique. Mais ce dernier passage permet aussi d'introduire le thème du vrai (ueram) et de l'imitation (pictam), une imitation si ressemblante (similem, similis) qu'il est difficile de la distinguer de l'original. On remarquera, à cet égard, l'emploi de ueram de préférence à uiuam, " vivante », employé ailleurs par Martial dans le cadre de l'éloge d'œuvres d'art, et dont la valeur métrique est la même ${ }^{83}$. Nous serions tentée de nous demander si l'on ne pourrait pas voir dans l'alternative finale une invitation à choisir entre deux interprétations de l'épigramme. Le lecteur pourrait ainsi considérer qu'Issa est réellement (uere) telle qu'elle est décrite et que le portrait qui en est fait est sincère (uerus) : c'est le choix d'une lecture encomiastique au premier degré. Mais l'option qui semble lui être suggérée par le second volet de l'alternative - on sait que l'on présente généralement en dernier lieu dans une disjonction la proposition à laquelle on donne la préférence et on connaît, en outre, l'importance de la place finale dans les épigrammes de Martial - est de considérer plutôt qu'Issa est embellie (picta) par cette description et que le portrait qui en est fait est lui-même l'objet d'une représentation (pictura) : Martial se livre à un pastiche satirique du portrait dithyrambique que Publius fait de sa chienne. Le dernier vers ou, plus précisément, le dernier mot - pictam - opèrerait ainsi un renversement, dégonflant les prétentions de Publius (le portrait d'Issa est flatté et sans ressemblance avec la réalité) en même temps qu'il indiquerait la désolidarisation ironique du sujet lyrique par rapport à l'éloge qui est fait de la chienne. Ce serait là finalement la pointe, mais ce n'est que dans le cadre d'une lecture satirique de l'épigramme qu'elle apparaît.

L'épigramme I, 109 ne brosse pas le portrait d'une chienne, mais d'un homme. Il s'agit de la satire d'un maitre qui fait preuve pour son animal de compagnie d'une complaisance ridicule ${ }^{84}$. Cette satire inclut une description encomiastique d'Issa, mais c'est là un pastiche des discours louangeurs de Publius; Martial, lui, se rattache à la tradition qui condamne ce type d'attitude. Les allusions érotiques, l'ironie qui perce à travers la naïveté et la disproportion des éloges, le caractère mimétique de certaines expressions relevant du style héroï-comique, de la facture et de la composition mêmes de la pièce, laissent transparaître l'intention satirique de l'épigrammatiste ${ }^{85}$, qui reste cependant discrète, au point de pouvoir n'être pas perçue ${ }^{86}$. L'ambiguïté inhérente à l'ironie, au pastiche, aux formes non explicites de discours rapporté - discours au style direct libre notamment - ouvre en effet la possibilité d'une lecture à deux niveaux. L'épigramme VII, 87, plus tardive et plus explicite ${ }^{87}$, où

\footnotetext{
${ }^{83}$ Martial emploie uiuit en III, 40 et uiuus en VI, 13.

${ }^{84}$ Comme l'ont souligné D. Sperber et D. Wilson, 1978, l'ironie est une attitude vis-à-vis de l'énoncé et de la personne à qui il faut l'attribuer (ici, Publius), et non vis-à-vis de l'objet de l'énoncé (en l'occurrence, Issa).

${ }^{85}$ L'ironie et l'imitation parodique constituent, comme on sait, deux des principaux procédés de la satire lorsque celle-ci s'applique aux discours du personnage visé.

${ }^{86}$ Que l'on se reporte, par exemple, aux différentes appréciations citées dans la note 2.

${ }^{87}$ Bien entendu, il n'est pas absolument impossible d'imaginer que, durant le laps de temps séparant la rédaction des deux poèmes, serait intervenue une dégradation des relations entre Martial et Publius, si tant est que ce dernier ait réellement existé. Nous n'avons aucun moyen de le vérifier. Mais ce qui reste, c'est, comme nous le soulignons, la nécessité de relire le premier poème de Martial et de le reconsidérer en tenant compte des
} 
Frédérique Fleck, «L'épigramme I, 109 de Martial : un poème encomiastique ou satirique ? » Article paru dans la Revue de philologie 82/1, 2008 [2010], p. 49-75.

Publius est mentionné dans une énumération d'amis de Martial atteints du même travers, lève en tout cas toute ambiguité et invite à relire à sa lumière, si besoin était, l'épigramme du livre I.

Frédérique FLECK

École normale supérieure (Paris)

dispositions d'esprit du poète qui se manifestent dans le poème plus tardif. C'est ce que nous avons entrepris de faire dans cet article. 